\title{
Symbolverzeichnis Band V
}

(alphabetisch)

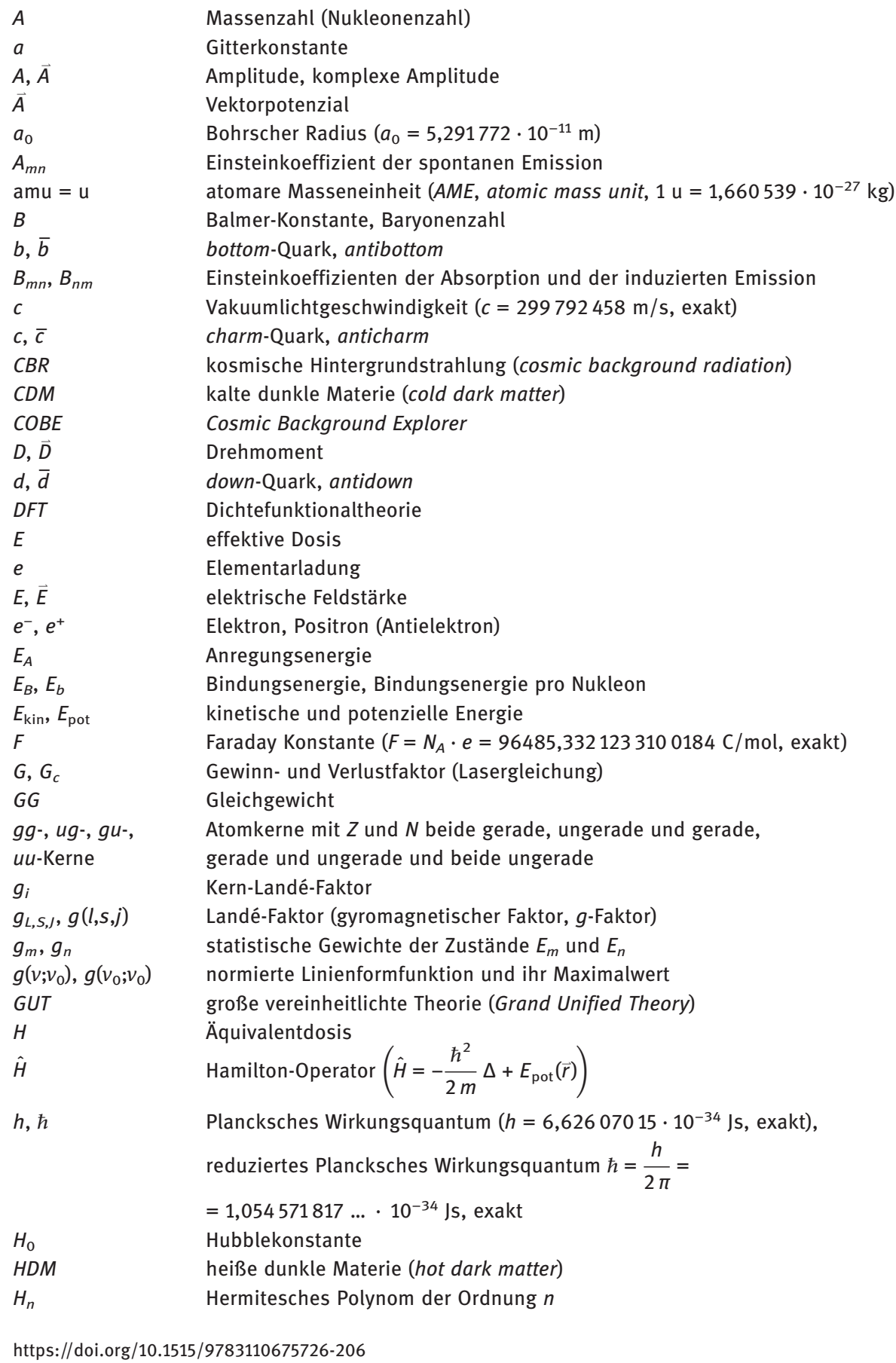




\section{$I, \vec{I}, \vec{i}$}

j

J, $\vec{\jmath}, J_{z}$

K

k

$k_{v}$

$k, \vec{k}$

$k f z$

$L$

$L, \vec{L}$

$\hat{\bar{L}}$

l

$l_{P}$

LHC

$L_{i}$

L-Welle

$L_{n+l}(\xi)$

MACHOS

$m_{e}, m_{n}, m_{p}$

$m_{j}$

$m_{l}$

$m_{p h}$

$m_{s}$

$N$

$N_{i}, N_{m}, N_{n}$

$n \equiv n(v) \equiv \bar{n}$

$n$

$N_{A}$

$n_{v}$

$O A M$

$P$

$p$

$p, \vec{p}, \hat{\bar{p}}, \vec{p}_{\text {rel }}$

$\hat{\bar{p}}, \hat{p}_{x}$

$P_{l}(\cos \theta)$

$p_{m}, \vec{p}_{m}$

$p_{r}, p_{\theta}, p_{\varphi}$

$P$-Welle

$Q$

$\hat{\bar{q}}$

$Q C D$
Intensität (Bestrahlungsstärke), elektrischer Strom, Isospin

(Elementarteilchen)

Eigendrehimpuls (Spin) der Nukleonen und des Atomkerns und Spinquantenzahl (Index $p, n$ oder ohne Index beim Kern)

Gesamtdrehimpulsquantenzahl

Gesamtdrehimpuls, z-Komponente des Gesamtdrehimpulses

Arbeit, Energie, integraler Absorptionskoeffizient, Kommutator

Boltzmannkonstante, Entartungsgrad eines Energiezustandes, Vermehrungs-

faktor (Kernreaktor)

Absorptionskoeffizient

Wellenzahl $\left(k=\frac{2 \pi}{\lambda}\right)$, Wellenvektor

kubisch-flächenzentriert

Länge des Laserresonators

Drehimpuls

Bahndrehimpulsoperator $(\hat{\bar{L}}=-i \hbar(\vec{r} \times \vec{\nabla}))$

Bahndrehimpulsquantenzahl (Nebenquantenzahl)

Planck-Länge

Large-Hadron-Collider (Großer Hadronen-Speicherring, Teilchenbeschleuniger am Europäischen Kernforschungszentrum CERN)

Leptonenzahl

linkszirkular polarisiertes Licht

Laguerresches Polynom

Massive Astrophysical Compact Halo Objects

Masse des Elektrons, des Neutrons, des Protons

magnetische Gesamtdrehimpulsquantenzahl

magnetische Bahndrehimpulsquantenzahl

Ruhemasse des Photons $\left(m_{p h}=0\right)$

magnetische Eigendrehimpulsquantenzahl (magnetische Spinquantenzahl)

Neutronenzahl $(N=A-Z)$, Anzahl der radioaktiven Kerne

Photonenzahl

mittlere Photonenzahl pro Schwingungsmode

Hauptquantenzahl (Energiequantenzahl), Neutron

Avogadro-Zahl $\left(6,02214076 \cdot 10^{23} \mathrm{~mol}^{-1}\right.$, exakt)

spektrale Modendichte

Bahndrehimpuls des Photons (orbital angular momentum)

Druck, Wahrscheinlichkeit, Wahrscheinlichkeitsdichte (H-Atom),

Laserleistung (ausgekoppelte Lichtleistung), Parität

Proton

Impuls, Viererimpuls, relativistischer Impuls (Raumanteil)

Impulsoperator $(\hat{\bar{p}}=-i \hbar \vec{\nabla})$, Impulsoperator in $x$-Richtung $\left(\hat{p}_{x}=-i \hbar \frac{\partial}{\partial x}\right)$

Legendresche Polynome

magnetisches Dipolmoment

kanonisch konjugierte Impulskomponenten (Sommerfeldsche Erweiterung des

Bohrschen Atommodells)

linear polarisiertes Licht

Ladung, Ladungsquantenzahl, Energie (Bindungsenergie, Reaktionswärme)

Viererimpuls

Quantenchromodynamik 


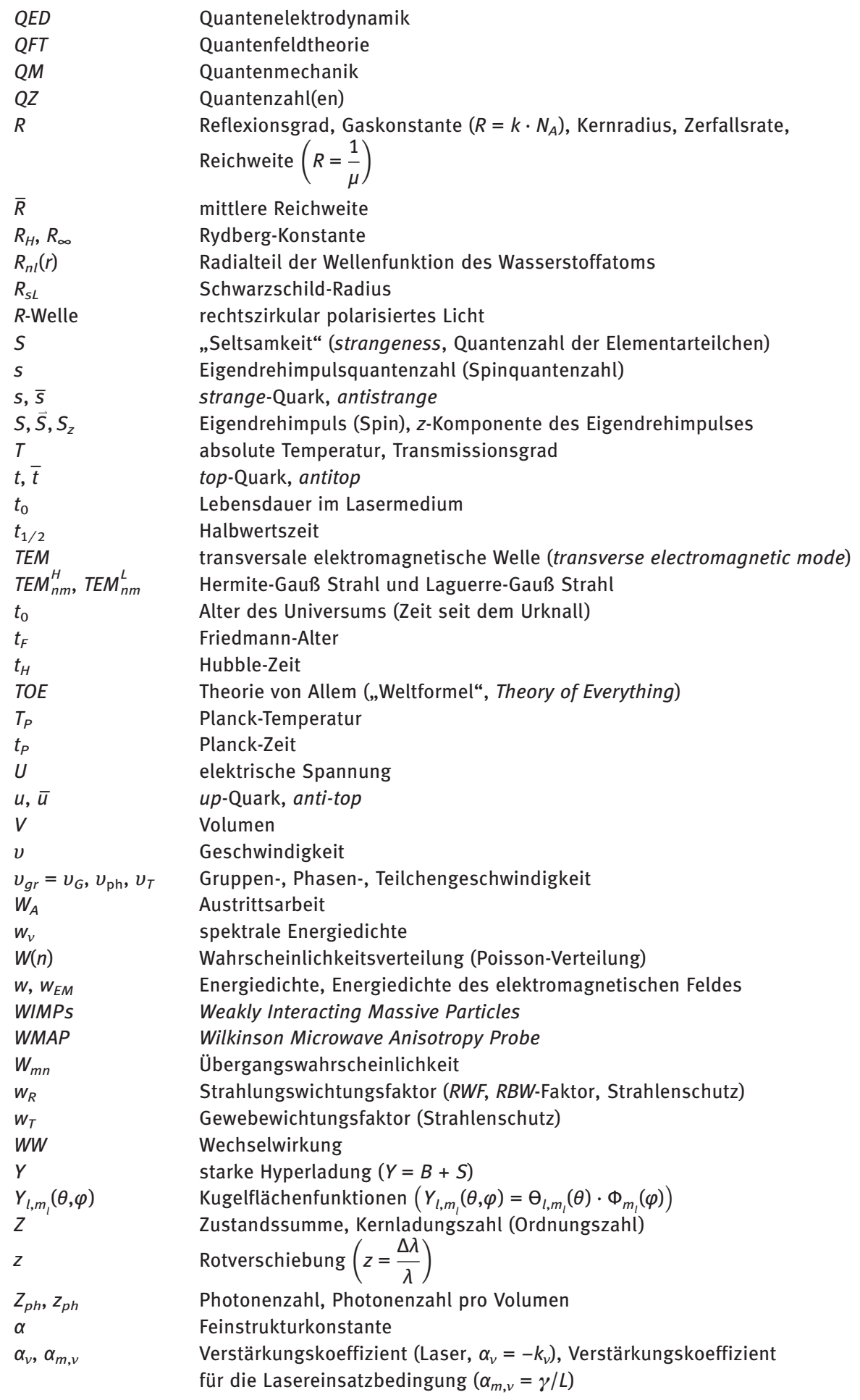


$$
\gamma
$$

$\gamma_{L}, \gamma_{S}$

$\Delta$

\section{$\Delta \lambda$}

\section{$\Delta M$}

$\Delta v_{G}, \Delta v_{L}$

$\Delta v_{\text {Mod }}$

$\varepsilon_{F}$

$\eta$

$\theta_{l, m_{l}}(\theta)$

$\Theta_{l m_{l}}(\theta), \Phi_{m_{l}}(\varphi)$

$\theta_{D}$

$\kappa$

$\Lambda$

$\hat{\Lambda}$

$\lambda$

$\mu$

$\mu^{-}, \mu^{+}$

$\mu_{B}$

$\mu_{e}^{L}, \vec{\mu}_{e}^{L}, \mu_{e}^{S}, \vec{\mu}_{e}^{S}, \mu_{e}^{\prime}, \vec{\mu}_{e}^{\prime}$

$\mu_{n}, \mu_{p}, \mu_{i}$
$v, \omega$
$v_{e}, v_{\mu}, v_{\tau}, \bar{v}_{e}, \bar{v}_{\mu}, \bar{v}_{\tau}$
$\rho$

$\rho_{c}, \rho_{u}$

$\rho_{P}$

$\sigma$

$\sigma^{-}-, \sigma^{+}$-Licht

$\sigma_{i}, \hat{\bar{\sigma}}$

$\bar{\tau}$

$\bar{\tau}_{\mathrm{sp}}$

$\tau^{-}, \tau^{+}$

$\tau_{E}$

$\Phi$

$\Phi_{A}$

$X^{+}, X^{-}$

$\psi, \psi(\vec{r}, t)$

$\psi(\vec{r})$
Halbwertsbreite (Spektrallinie)

Verlustfaktor (Laser), Lorentz-Faktor $\left(\gamma=\frac{1}{\sqrt{1-v^{2} / c^{2}}}=\frac{1}{\sqrt{1-\beta^{2}}}\right)$

gyromagnetisches Verhältnis $\left(\gamma_{L}=\frac{\left|\vec{\mu}_{e}^{L}\right|}{|\vec{L}|}, \gamma_{S}=\frac{\left|\vec{\mu}_{e}^{S}\right|}{|\vec{S}|}\right)$

Laplace-Operator $\left(\Delta=\frac{\partial^{2}}{\partial x^{2}}+\frac{\partial^{2}}{\partial y^{2}}+\frac{\partial^{2}}{\partial z^{2}}\right)$

Compton-Verschiebung

Massendefekt

Halbwertsbreiten für Gauß- und Lorentz-Linienprofil

Longitudinalmodenabstand

Fermienergie

Wirkungsgrad

zugeordnete Kugelfunktionen (zugeordnete Legendresche Polynome,

$\Theta_{l, m_{l}}(\theta)=P_{l}^{\left|m_{l}\right|}(\cos \theta)$

Winkelanteile der Wellenfunktion des Wasserstoffatoms

Debye-Temperatur

integrierter Absorptionsquerschnitt pro Atom

kosmologische Konstante

Legendrescher Operator $\left(\hat{\Lambda}=-\left[\frac{1}{\sin \theta} \frac{\partial}{\partial \theta}\left(\sin \theta \frac{\partial}{\partial \theta}\right)+\frac{1}{\sin ^{2} \theta} \frac{\partial^{2}}{\partial \varphi^{2}}\right]\right)$

Wärmeleitfähigkeit, Wellenlänge, Zerfallskonstante (Zerfallswahrscheinlichkeit) reduzierte Masse $\left(\mu=\frac{m M}{m+M}\right)$, Absorptionskoeffizient

Myon, Antimyon

Bohrsches Magneton

Gesamtdrehimpulsmoment des Elektrons

magnetisches Moment der Nukleonen und des Atomkerns

Frequenz, Kreisfrequenz $(\omega=2 \pi v)$

Elektron-, Myon-, Tauonneutrino und ihre Antiteilchen

elektrische Ladungsdichte

kritische und tatsächliche Massendichte des Universums

Planck-Dichte

elektrische Leitfähigkeit, Wirkungsquerschnitt

rechtszirkular ( $R$-Zustand), linkszirkular polarisiertes Licht (L-Zustand)

Pauli-Spinmatrizen

mittlere Lebensdauer, mittlere Stoßzeit (Lorentz-Linienprofil)

mittlere Lebensdauer bei spontaner Emission

Tauon, Antitauon

Einschlusszeit (Fusionsreaktor)

Strahlungsleistung, elektrostatisches Potenzial

Austrittspotenzial

Spin(eigen)funktion $\left(X^{+}=X^{\uparrow}=\left(\begin{array}{l}1 \\ 0\end{array}\right), X^{-}=x^{\downarrow}=\left(\begin{array}{l}0 \\ 1\end{array}\right)\right)$

Materiewellen-Wellenfunktion (Wahrscheinlichkeitsamplitude)

(nur) ortsabhängige Wellenfunktion 
$\Omega_{m}$

$\omega_{L}, \vec{\omega}_{L}$
Dichteparameter (Verhältnis der tatsächlichen zur kritischen Massendichte des Universums) mit $\Omega_{m}=\Omega_{b}+\Omega_{d}+\Omega_{\Lambda}\left(\Omega_{b} \ldots\right.$ sichtbare „baryonische“ Materie, $\Omega_{d} \ldots$ Dunkle Materie, $\Omega_{\wedge} \ldots$. Dunkle Energie)

Larmorfrequenz, Larmor-Winkelgeschwindigkeit

\section{Wichtige physikalische Größen, Band V}

Elementarladung
Lichtgeschwindigkeit
Boltzmannkonstante

Avogadro-Zahl

Plancksches

Wirkungsquantum

reduziertes Plancksches

Wirkungsquantum

Compton-Wellenlänge

des Elektrons

Masse des Elektrons

Na-D Linie (NIST Database)

Energieumrechnung

Wellenzahl-Energie-

Äquivalent

Faraday-Konstante

atomare Masseneinheit $(\mathrm{amu}) 1 \mathrm{u}=(1,66053906660 \pm 0,00000000050) \cdot 10^{-27} \mathrm{~kg}=$

Rydberg-Konstante

Feinstrukturkonstante

Kehrwert

Bohrscher Radius

Bohrsches Magneton

Magnetisches Moment des Elektrons $=(931,49410242 \pm 0,0000000028) \mathrm{MeV} / \mathrm{c}^{2}$

$$
\begin{aligned}
e & =1,602176634 \cdot 10^{-19} \mathrm{C}, \text { exakt } \\
c & =299792458 \mathrm{~m} / \mathrm{s}, \text { exakt } \\
k & =\frac{R}{N_{A}}=1,380649 \cdot 10^{-23} \mathrm{~J} \cdot \mathrm{K}^{-1}= \\
& =1,380649 \cdot 10^{-23} \mathrm{eV} / 1,602176634 \cdot 10^{-19} \mathrm{~K}, \text { exakt }= \\
& =8,617333262 \ldots \cdot 10^{-5} \mathrm{eVK}^{-1} \\
N_{A} & =6,02214076 \cdot 10^{23} \mathrm{~mol}^{-1}, \text { exakt } \\
h & =6,62607015 \cdot 10^{-34} \mathrm{Js}= \\
& =6,62607015 \cdot 10^{-34} / 1,602176634 \cdot 10^{-15}, \text { exakt }= \\
& =4,135667696 \cdot 10^{-15} \mathrm{eV} \mathrm{s}
\end{aligned}
$$

$$
\begin{aligned}
\hbar & =\frac{h}{2 \pi}=1,054571817 \ldots \cdot 10^{-34} \mathrm{Js}= \\
& =6,582119569 \ldots \cdot 10^{-16} \mathrm{eV} \mathrm{s}, \text { exakt }
\end{aligned}
$$

$$
\begin{aligned}
\lambda_{C} & =(2,42631023867 \pm 0,00000000073) \cdot 10^{-12} \mathrm{~m} \\
m_{e} & =(9,1093837015 \pm 0,0000000028) \cdot 10^{-31} \mathrm{~kg}= \\
& =(0,51099895000 \pm 0,00000000015) \mathrm{MeV} / \mathrm{c}^{2}= \\
& =(5,48579909065 \pm 0,00000000016) \cdot 10^{-4} \mathrm{u}
\end{aligned}
$$

$\lambda_{D 1}=(589,592424 \pm 0,000003) \mathrm{nm}$,

$\lambda_{D 2}=(588,995095 \pm 0,000003) \mathrm{nm}$

$1 \mathrm{eV}=1,602176634 \cdot 10^{-19} \mathrm{~J}$, exakt

$1 \mathrm{~J}=6,241509074 \ldots \cdot 10^{18} \mathrm{eV}$, exakt

$\frac{1}{\mathrm{~m}} \cdot \mathrm{h} \cdot \mathrm{c}=1,239841984 \ldots \cdot 10^{-6} \mathrm{eV}$

$F=N_{A} \cdot e=96485,3321233100184 \mathrm{C} / \mathrm{mol}$, exakt

$R_{\infty}=(1,0973731568160 \pm 0,0000000000021) \cdot 10^{7} \mathrm{~m}^{-1}$

$\alpha=(7,2973525693 \pm 0,0000000011) \cdot 10^{-3}$

$\alpha^{-1}=137,035999084 \pm 0,000000021$

$a_{0}=(0,529177210903 \pm 0,000000000080) \cdot 10^{-10} \mathrm{~m}$

$\mu_{B}=(9,2740100783 \pm 0,0000000028) \cdot 10^{-24} \mathrm{JT}^{-1}=$

$=(5,7883818060 \pm 0,0000000017) \cdot 10^{-5} \mathrm{eVT}^{-1}$

$$
\begin{aligned}
\left|\mu_{e}^{S_{z}}\right| & =9,2847647043 \pm 0,0000000028) \cdot 10^{-24} \mathrm{JT}^{-1}= \\
& =(1,00115965218128 \pm 0,00000000000018) \mu_{B}
\end{aligned}
$$


Landé-Faktor ( $g$-Faktor) für den Spin des Elektrons

Kernmagneton

$$
\begin{aligned}
g_{S} & =g=2,00231930436256 \pm 0,00000000000035 \\
\mu_{N} & =(5,0507837461 \pm 0,0000000015) \cdot 10^{-27} \mathrm{JT}^{-1}= \\
& =(3,15245125844 \pm 0,00000000096) \cdot 10^{-8} \mathrm{eVT}^{-1}
\end{aligned}
$$

Proton:

Masse

magnetisches Moment

Neutron:

Masse

magnetisches Moment

Alter des Universums

Hubble Konstante

(Planck Teleskop)

$$
\begin{aligned}
m_{p} & =(1,007276466621 \pm 0,000000000053) \mathrm{u}= \\
& =(938,27208816 \pm 0,00000029) \mathrm{MeV} / \mathrm{c}^{2}= \\
& =(1,67262192369 \pm 0,00000000051) \cdot 10^{-27} \mathrm{~kg} \\
\mu_{p} & =(2,79284734463 \pm 0,00000000082) \mu_{N}= \\
& =(1,41060679736 \pm 0,00000000060) \cdot 10^{-26} \mathrm{JT}^{-1}
\end{aligned}
$$

$$
\begin{aligned}
m_{n} & =(1,00866491595 \pm 0,00000000049) \mathrm{u}= \\
& =(939,56542052 \pm 0,00000054) \mathrm{MeV} / \mathrm{c}^{2}= \\
& =(1,67492749804 \pm 0,00000000095) \cdot 10^{-27} \mathrm{~kg}
\end{aligned}
$$$$
\mu_{n}=(-1,91304273 \pm 0,00000045) \mu_{N}=
$$$$
=(-0,96623651 \pm 0,00000023) \cdot 10^{-26} \mathrm{JT}^{-1}
$$

$t_{0}=(13,787 \pm 0,020) \cdot 10^{9}$ Jahre

$H_{0}=(67,4 \pm 0,5)(\mathrm{km} / \mathrm{s}) / \mathrm{Mpc}$ 